J Child Adolesc Psychopharmacol Vol. 11(4):377-388 (2001)

ISSN: (print 1044-5463) (online 1557-8992)

doi: 10.1089/104454601317261555

This is a peer reviewed pre-print version of the following article: Methodological Issues in Designing a Multisite

Trial of Risperidone in Children and Adolescents with Autism, which has been published in final form at:

http://www.liebertpub.com

http://www.liebertpub.com/cap

http://www.liebertonline.com/doi/pdfplus/10.1089/104454601317261555

(C) 2001 Mary Ann Liebert, Inc. Publishers

\title{
Methodological Issues in Designing a Multisite Trial of Risperidone in Children and Adolescents with Autism
}

Larry Scahill M.S.N., Ph.D., Yale University, New Haven, Connecticut, James McCracken, M.D., University of California Los Angeles, Los Angeles, California, Christopher J. McDougle, M.D., Indiana University, Bloomington, Indiana, Michael Aman, Ph.D., Ohio State University, Columbus, Ohio, L. Eugene Arnold, M.Ed, M.D., Ohio State University, Columbus, Ohio, Elaine Tierney, M.D., Kennedy Krieger Institute, Baltimore, Maryland Pegeen Cronin, Ph.D., University of California Los Angeles, Los Angeles, California, Mark Davies, M.P.H., Columbia University, New York, New York., Jaswinder Ghuman, M.D., Kennedy Krieger Institute, Baltimore, Maryland, Nilda Gonzalez, M.D., Kennedy Krieger Institute, Baltimore, Maryland, Kathleen Koenig, M.S.N., Yale University, New Haven, Connecticut, Ronald Lindsay, M.D., Ohio State University, Columbus, Ohio, Andres Martin, M.D., Yale University, New Haven, Connecticut, James McGough, M.D., University of California Los Angeles, Los Angeles, California, David J. Posey, M.D., Indiana University, Bloomington, Indiana, Naomi Swiezy, Ph.D., Indiana University, Bloomington, Indiana, Fred Volkmar, M.D., Yale University, New Haven, Connecticut, Louise Ritz, M.B.A., National Institute of Mental Health, Bethesda, Maryland., Benedetto Vitiello, M.D. National Institute of Mental Health, Bethesda, Maryland.

Objective: To describe the methodological challenges and decisions made in developing a multisite, controlled study of risperidone in children and adolescents with autism.

Methods: Review the design considerations for clinical trials in children with autistic disorder accompanied by severe tantrums, aggressive and/or self-injurious behaviors. These design considerations include the definition of inclusion criteria that are relevant to clinical practice and matching study design to the goal of evaluating short- and long-term effects. Additional ethical and scientific issues concern the length of trial and sample size.

Results: We undertook a short-term, placebo-controlled study to evaluate the efficacy and safety of risperidone in children and adolescents with autistic disorder. This trial design was followed by an extended open-label maintenance on risperidone to confirm durability of treatment effects and to monitor safety. Finally, a placebo-controlled discontinuation study tested the need for continuous treatment.

Conclusions: In the absence of standard pharmacological treatment for children with autistic disorder, a placebo-controlled study remains the most appropriate method of testing efficacy and safety. The clinical relevance of this study is enhanced by the addition of an extended maintenance phase followed by a placebo discontinuation.

\section{Introduction}

Autistic disorder is a chronic condition of early childhood onset characterized by profound impairments in social relatedness, communication skills, and restricted patterns of 
behavior affecting 5-20 persons per 10,000 in the school-age population (Fombonne 1999; Chakrabarti and Fombonne 2001). Other behaviors, such as hyperactivity, self-injurious behavior (SIB), aggression toward others, extreme intolerance of change, and/or stereotypies frequently complicate clinical management. Although there is currently no cure for autism and other pervasive developmental disorders (PDDs), the appropriate use of medication to control behavioral dysregulation may enhance the individual's function and ability to benefit from educational and behavior modification interventions. Recent surveys indicate that a wide range of medications is used in the treatment of children and adolescents with autism and other PDDs (Aman et al. 1995; McDougle et al. 2000). Empirical support for most pharmacological treatments, however, is limited.

In 1997 the National Institute of Mental Health (NIMH) awarded competitive contracts to establish a network of Research Units on Pediatric Psychopharmacology (RUPP) for autism and PDDs. The impetus for this NIMH initiative was the recognition that psychopharmacological research in autism and other PDDs is marked by inconsistent findings in small samples that fail to guide clinical practice. As a first project, the five-site network agreed to conduct a study of risperidone in children and adolescents with autism. The primary aims of the study were to evaluate the short-term efficacy and safety of risperidone in children with autistic disorder and to determine whether observed clinical benefits and safety were stable over time. Methodological challenges included identifying the target symptoms in a sample of subjects with severe developmental disorders, defining inclusion criteria that are pertinent to clinical practice, matching study design to evaluate both short- and long-term effects, and characterizing subjects in order to identify predictors of treatment response.

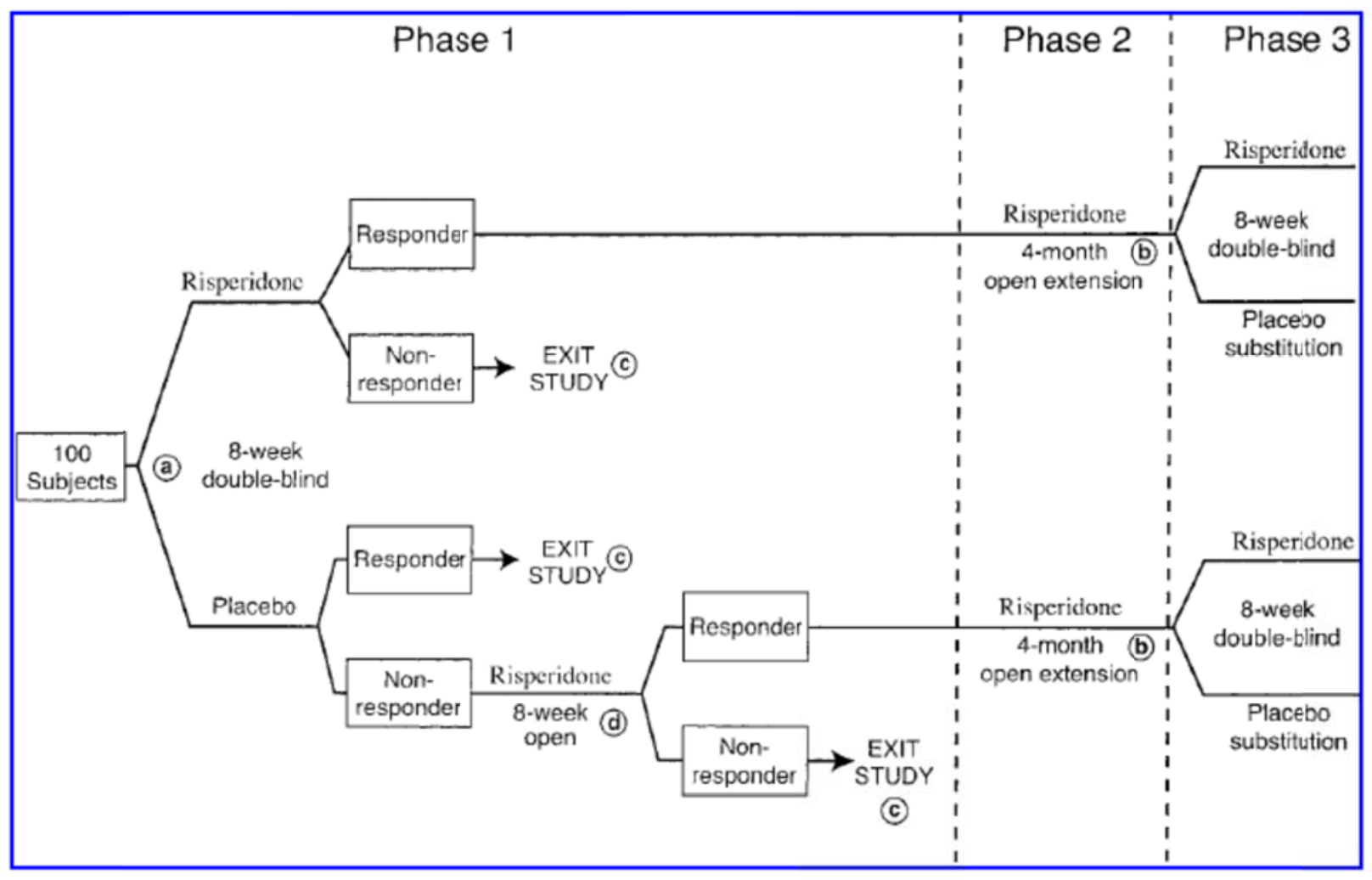

Fig. 1. A schematic diagram of study design.

$\mathrm{a}=$ random assignment to risperidone or placebo; 
$\mathrm{b}=$ random assignment to continued treatment with risperidone or gradual placebo substitution;

$\mathrm{c}=$ continued observation or treatment as appropriate;

$\mathrm{d}=$ uses same dose and visit schedule as in double-blind phase.

The study was divided into three phases designed to evaluate the short- and long-term safety and efficacy of risperidone in children and adolescents with autistic disorder. Phase 1 was an 8-week, double-blind, randomized, parallel-groups comparison of risperidone and placebo. Subjects assigned to placebo who did not improve were offered an 8-week open-label trial of risperidone. Phase 2 consisted of a 4-month open-label treatment with risperidone for patients who showed improvement in the 8-week acute trial. The subsequent phase 3 was a randomized, double-blind, placebo-controlled discontinuation study (see Fig. 1). The target symptoms included tantrums, aggression, SIB, agitation, screaming, emotional rigidity, and repetitive behavior as measured by the Irritability subscale of the Aberrant Behavior Checklist completed by the parent or primary caretaker (Aman and Singh 1986; Aman et al. 1985; Mashburn and Arman 1992). This subscale was selected because of its favorable psychometric properties (Mashburn and Aman 1992), and it contains the behaviors of interest. To avoid overreliance on an informant-based measure, the study also included clinician and laboratory measures as secondary outcomes (for a detailed description of assessment measures used in the study, see Arnold et al. 2000). The rationale for choosing risperidone was based on concern about the shortand long-term side effects of haloperidol (Campbell et al. 1997) and emerging pilot data from several centers showing encouraging results with risperidone (see McDougle et al. 2000 for a detailed review). This article describes the methodological issues encountered and the design decisions taken in developing the multisite clinical trial of risperidone by the RUPP Autism Network.

\section{Short-Term Safety and Efficacy}

The primary purpose of the first phase of the study was to compare the short-term safety and efficacy of risperidone and placebo in the treatment of children and adolescents with autistic disorder. We hypothesized that risperidone would be more effective than placebo in reducing impulsive aggression, tantrums, SIB, screaming, and out-of-control behavior associated with autistic disorder. Important exploratory questions of interest included whether risperidone (a) would be more effective than placebo for improving repetitive behavior in children and adolescents with autistic disorder; (b) would be associated with extrapyramidal side effects; (c) would be associated with specific side effects such as excessive weight gain or prolactinemia; and (d) whether specific CYP450 2D6 genotypes would be associated with clinical response, a range of adverse effects, and/or higher steady-state levels of risperidone.

\section{Placebo control}

The rationale for placebo control was based on the intent to evaluate safety and efficacy. First, placebo response has been observed in serious disorders such as autism (Sandler et al. 1999). Second, the only medication that could be proposed as a standard treatment for aggression and SIB in children with autistic disorder is haloperidol (Anderson et al. 1989). However, clinicians and families often avoid using this medication due to legitimate concerns about shortand long-term side effects (Campbell et al. 1977; McDougle et al. 2000). A direct comparison of risperidone or haloperidol was discarded as clinically unacceptable. Behavior modification treatment was not considered a reasonable comparison either, because it is often tried before pharmacotherapy and it would be difficult to ensure blinding in the study. In the absence of a satisfactory standard treatment, therefore, placebo control was fully justified. 
Placebo control was also justified because autism is a chronic condition, and treatment of associated behavioral symptoms often requires pharmacotherapy for long periods of time. It is therefore important to provide clear-cut evidence of medication efficacy and safety in order to validate extended use. A placebo-controlled trial is the most efficient method of testing treatment effects. Moreover, the placebo control group provides a contrast group for assessment of adverse events. The decision to conduct an 8-week trial was a compromise between the amount of time required to show a treatment effect and our interest in avoiding a prolonged period without active treatment for subjects randomly assigned to placebo. To minimize the potential burden of placebo assignment, subjects who were randomized to placebo and failed to show a clinically meaningful response (placebo nonresponders) were eligible to participate in an 8-week openlabel trial of risperidone after a minimum of 4 weeks in the double-blind phase (see Fig. 1). The 8-week, open-label trial mimicked the double-blind treatment with respect to dose schedule, frequency of follow-up visits, and outcome measurement.

\section{Sample selection}

Tables 1 and 2 present the inclusion and exclusion criteria used in the study. Selection of these criteria was guided by the goal of producing generalizable results. For example, $25 \%$ to $33 \%$ of children with autism also have seizures (Cohen and Volkmar 1997). If risperidone proves to be safe and effective in autism, children on anticonvulsants are likely to be treated with risperidone. Failure to include subjects on anticonvulsants would have limited the clinical application of the study findings. On the other hand, changes in anticonvulsant dose during the study could have had an impact on the child's behavior and/or the interpretation of adverse effects. Thus, this entry criterion was qualified by insisting that both the dose of medication and the seizure disorder were stable prior to entry.

The decision to include only subjects diagnosed with autism was based on the presumed greater variability in response if a wider range of PDD subjects was included in the study. Although autism is unlikely to be a homogeneous disorder, the inclusion of children with other forms of PDD in the study invited undue heterogeneity. Within the diagnostic category of autism, however, we attempted to maximize generalizability by including subjects across a wide range of IQ. Children below a mental age of 18 months were excluded because of the fundamental problem of making the diagnosis of autistic disorder in profoundly retarded children (Lord et al. 1994).

The decision to include only children diagnosed with autistic disorder had two important implications. First, the prevalence of autistic disorder is 5 to 20 cases per 10,000 compared to 10 to 40 cases per 10,000 for other forms of PDD (Fombonne 1999; Chakrabarti and Fombonne 2001). Thus, restricting study participation to subjects with autism accompanied by severe behavioral dyscontrol reduced the pool of eligible subjects. Second, although each site had investigators with expertise in the assessment and treatment of autism, the decision to include subjects with autism required a network-wide training on the administration of the Autism Diagnostic Interview-Revised to ensure reliability in the diagnosis.

\section{Randomization and dose schedule}

To make certain that treatment groups would be comparable, the randomization was balanced within site by pubertal status (Tanner stages I and II for prepubertal status and Tanner III or higher for postpubertal status), gender, and anticonvulsant use. The medication schedule was based on the subject's weight. Children weighing at least $20 \mathrm{~kg}$ at study entry were started at a dose of $0.5 \mathrm{mg}$ at bedtime. As shown in Table 3 (top half), the dose was increased to $0.5 \mathrm{mg}$ 
twice daily at day 4 and gradually thereafter to a maximum of $2.5 \mathrm{mg}$ per day $(1.0 \mathrm{mg}$ in the morning and $1.5 \mathrm{mg}$ at bedtime) by day 29. A similar but slightly faster dose schedule was used for children weighing $45 \mathrm{~kg}$ or more (maximum of $1.5 \mathrm{mg}$ in the morning and $2.0 \mathrm{mg}$ at bedtime) as is shown in Table 3 (bottom half). For children with baseline weight between 15 and $20 \mathrm{~kg}$, the starting dose was $0.25 \mathrm{mg} / \mathrm{day}$.

- $\quad$ Males and females between the ages of 5 years and 17 years 2 months

- DSM-IV diagnosis of autistic disorder (established by clinical assessment, corroborated by the Autism Diagnostic Interview)

- Inpatients or outpatients

- Medication free for at least 2 weeks for all psychotropic medications (4 weeks for fluoxetine or depot neuroleptics)

- Anticonvulsants used for the treatment of a seizure disorder were permitted if the dosage had been stable for 4 weeks and the patient had been seizure free for at least 6 months.

- Clinical Global Impressions severity score of at least 4 (moderately ill) at baseline rated by the blinded rater

- A score of 18 or greater on the Irritability subscale of the Aberrant Behavior Checklist at baseline (on the parent-rated and/or clinician-rated version)

- A mental age of at least 18 months as measured by the age-appropriate form of the Wechsler Intelligence Test, by the revised Leiter, or by the Mullen

Table 1. Inclusion Criteria for Double-Blind Risperidone Study in Children and Adolescents with Autism

- Females with a positive Beta human chorionic gonadotropin (HCG) pregnancy test

- Evidence of a prior adequate trial with risperidone (defined as duration of 2 weeks or more at a dose of at least $1 \mathrm{mg}$ /day)

- Evidence of hypersensitivity to risperidone (defined as allergic response [e.g., skin rash] or potentially serious adverse effect [e.g., significant tachycardia])

- Past history of neuroleptic malignant syndrome

- DSM-IV diagnosis of schizophrenia, another psychotic disorder, or substance abuse

- A significant medical condition such as heart disease, hypertension, liver or renal failure, or pulmonary disease identified by history, physical examination, or laboratory tests

- Weight less than $15 \mathrm{~kg}$

Table 2. Exclusion Criteria for Double-Blind Risperidone Study

In all cases, the dose schedule was not fixed. The decision to increase the dose above 1 mg/day was based on clinical judgment. For example, in the presence of a positive clinical response as evidenced by a rating of much improved or very much improved on the Global Improvement item of the Clinician's Global Impressions (CGI) scale or clinically important side effects (e.g., sedation), the dosage was not automatically increased. If a symptomatic deterioration was evident at a subsequent visit, or if the dose-limiting side effect resolved, the clinical team was free to resume upward dose adjustment. In the double-blind trial, there were no dose increases allowed after day 29. After day 29, the dose was maintained until the endpoint (day 56), unless adverse effects emerged at which point the primary clinician could lower the dose of the medication. Finally, the clinical team was permitted to prescribe benztropine to manage extrapyramidal symptoms.

\begin{tabular}{lcc}
\hline \multicolumn{3}{l}{ Model dose schedule for subjects between 20 and $45 \mathrm{~kg}$} \\
\hline $\begin{array}{l}\text { Morning dose } \\
\text { (mg) }\end{array}$ & $\begin{array}{l}\text { Bedtime dose } \\
(\mathrm{mg})\end{array}$ \\
\hline 1 & $\overline{0}$ & 0.5 \\
4 & 0.5 & 0.5 \\
8 & 0.5 & 1.0
\end{tabular}




\begin{tabular}{lcc}
16 & 1.0 & 1.0 \\
22 & 1.0 & 1.5 \\
$29^{\mathrm{a}}$ & 1.0 & 1.5 \\
\hline \multicolumn{2}{l}{ Dose schedule for subjects $\geq 45 \mathrm{~kg}$} & \\
\hline 1 & $\overline{0.5}$ & 0.5 \\
4 & 0.5 & 0.5 \\
8 & 1.0 & 1.0 \\
12 & 1.0 & 1.0 \\
15 & 1.5 & 1.5 \\
18 & 1.5 & 1.5 \\
23 & 1.5 & 2.0 \\
$29^{\mathrm{a}}$ & & 2.0 \\
\hline
\end{tabular}

Table 3. Dose Schedule

${ }^{\mathrm{a}}$ Visit on day 29 was last point to authorize a dose increase; dose reduction to manage side effects can be done at any time.

Compliance.

Parents were given a diary card at each visit with day-by-day instructions about the medication dose. Compliance was monitored by pill counts and by review of the diary card at each visit. Any deviation from the prescribed dose was discussed in order to ensure an optimal dosing strategy. If the compliance fell below $70 \%$ of the prescribed dosage for a given week via pill count, parents were educated about the importance of taking the medication as prescribed. Continued deviation from the prescribed dosing regimen, in the absence of extenuating circumstances, would lead to the subject's termination from the study. Due to the predictable difficulties of drawing blood samples from this nonverbal, often aggressive population, we did not include the measurement of plasma drug level determinations as an additional method of discerning compliance during the study. The drug level drawn at endpoint, which was coincident with blood sampling for laboratory tests, permitted a check on compliance and an examination of the relationship between CYP 2D6 genotype, blood level, and clinical response.

\section{Ensuring blindness and breaking the blind}

The blind in a placebo-controlled study may be compromised by the emergence of obvious side effects. Therefore, each subject was followed by two clinicians: a blinded clinical evaluator, who focused on clinical ratings, and a blinded primary clinician, who evaluated side effects and adjusted the medication dosage. At the end of the double-blind phase, but before the treatment code was broken, each subject was classified as a responder or nonresponder. Positive treatment response was defined by a decrease of $25 \%$ on the Irritability subscale of the Aberrant Behavior Checklist and a rating of much improved or very much improved on the Global Improvement item on the CGI at endpoint. Once this determination was made, the primary clinician broke the treatment code and initiated the next step in the subject's treatment. The rationale for breaking the blind was to ensure continuity of care for subjects who benefited from the active medication during the double-blind phase and to evaluate subsequent clinical response to open-label treatment for placebo nonresponders in the double-blind phase.

At the end of the double-blind phase, subjects were cross-classified by treatment assignment and clinical response (risperidone responder, risperidone nonresponder, placebo nonresponder, or placebo responder). Risperidone responders were invited to participate in the 4month extension study (Fig. 1). These subjects were seen monthly for 4 months (see description 
of phase 2 below). Although visits were scheduled on a monthly basis, interim visits with the primary clinician were scheduled on an as needed basis.

Placebo nonresponders entered an 8-week, open-label risperidone trial, which mimicked the double-blind study with respect to weekly visits and dose schedule. These subjects were seen weekly for the first 4 weeks by the primary clinician to monitor side effects and adjust the dosage. The blinded clinical evaluator did not resume clinical ratings until the end of the first month in the open-label phase. This plan ensured continuity in that the same blinded clinical evaluator conducted outcome measures. It also provided some additional protection of the blind in that the blinded clinical evaluator remained unaware of treatment assignment during the double-blind study. Subjects who were judged to be responders after 8 weeks of open-label treatment were invited to enroll in the 4-month extension (phase 2) followed by the placebocontrolled discontinuation phase.

Risperidone nonresponders were treated in accordance with the best judgment of the clinical team and the family's preference. Similarly, responders to placebo in the double-blind phase were not invited into the 8-week open-label study (Fig. 1). These subjects were followed by the clinical team, and treatment decisions were based on the subject's clinical picture.

\section{Data analytic plan for primary hypothesis in phase 1}

Sample size.

The sample size of 100 was chosen to ensure sufficient power to detect a moderate treatment difference between risperidone and placebo. Based on conservative estimates, such as a positive response of $40 \%$ for the risperidone group and $10 \%$ for the placebo group, the power to detect a difference between treatments is $94 \%$ with 50 in each group. Even if the difference in the rate of positive response is only $25 \%$, the power to detect that difference between groups would be $86 \%$ at the conventional $5 \%$ level of significance (Borenstein et al. 1997). The sample size of 100 was also intended to identify differences in the frequency of common adverse events across the two treatments. Adverse events with an expected frequency of $6 \%$ or larger are detectable with the projected sample size of 50 in each group with a power of $95 \%$, whereas a frequency of $3 \%$ or larger is detectable with a power of $78 \%$.

\section{Evaluation of efficacy.}

The intent-to-treat principle in which all randomized subjects are included in the analyses will guide the evaluation of efficacy (Fleiss 1986). The primary outcome measure, the Irritability subscale of the Aberrant Behavior Checklist (completed by the parent), will be analyzed with a mixed effects linear model, also called a random regression model (Gibbons et al. 1993). In this approach, each subject's response during the trial is modeled by regressing the response against time. The intercept and slope of the regression are estimated for each subject. A subject who shows a strongly positive response over time will have a much larger regression slope than a subject who barely changes over time. The average slope of the regression of response on time is compared across the risperidone and placebo groups and tested for statistical significance. An obvious advantage of the mixed model is that it permits a careful look at individual responses as well as group effects.

Another advantage of the mixed model is that it is more tolerant of missing data than repeated-measures analysis of variance, which only uses those subjects who have complete data. Analyses of data from only those subjects who complete the protocol may be biased by factors related to attrition. By contrast, subjects who terminate the protocol prematurely can be included 
in the mixed model. The random regression model can estimate change parameters as long as there are at least two observations over time.

A more straightforward and clinically relevant approach to evaluating the efficacy of risperidone is to calculate the difference in the rate of positive response between the two treatment groups. By using the definition of positive response described above, we will also compare the rate of positive response in each treatment group by a chi-square test. This computation will provide clinicians with an estimate of how likely a patient with similar problems will respond to treatment with risperidone.

Evaluation of safety.

Adverse events were systematically assessed and recorded at each visit by the primary clinician. Height, weight, vital signs, and concomitant medications were also documented at each weekly visit. An electrocardiogram and routine laboratory studies were conducted at the baseline and final visit in the double-blind phase (Kumra et al. 1997). Because our interest was in documenting any changes in cardiac conduction, electrocardiograms were read by pediatric cardiologists at all sites. Laboratory measures included complete blood count, electrolytes, blood urea nitrogen, creatinine, liver function tests, and urinalysis. Electroencephalograms were not done.

Differences in rates of adverse events between the risperidone and placebo groups will be tested by chi-square with Fisher's exact test when cell sizes are less than 5. Other analytic approaches will compare counts of adverse events in each of the two treatment groups using $t$ tests.

\section{Cytochrome P450 2D6 (CYP2D6) enzyme genotype and clinical response.}

Risperidone is well absorbed orally and has an estimated 4-hour half-life in adults. The major metabolic pathway is oxidation via the CYP2D6 pathway (Heykants et al. 1994). The major metabolite, 9-hydroxyrisperidone, appears to be equally potent as risperidone with respect to receptor binding activity. However, it has a 17-hour half-life and is not dependent on CYP2D6.

Recent studies indicate that $5 \%$ to $10 \%$ of the Caucasian population and $2 \%$ to $6 \%$ of the African American population have little or no activity of the CYP2D6 enzyme (He et al. 1999; Poolsup et al. 2000). This lack of CYP2D6 activity is inherited as an autosomal recessive trait (homozygous for the low activity allele). By contrast, a small percentage of the population are ultrarapid metabolizers of CYP2D6 substrates due to gene amplification of the normal allele. To date, no studies have examined the relationship of CYP2D6 genotypes to risperidone level or clinical response in children and adolescents. By design, the dose of risperidone in children and adolescents in this study was constrained to fall in a narrow range (roughly $1.5 \mathrm{mg}$ to $3.5 \mathrm{mg}$ per day in divided doses). Despite this narrow range, we plan to evaluate the relationship between serum drug level, clinical response, and 2D6 genotype. The first set of exploratory analyses will examine the correlation of 2D6 genotype and blood level (adjusted for oral dose and body weight).

Given that 9-hydroxyrisperidone is not dependent on CYP2D6, individuals with low activity of CYP2D6 are likely to show a higher ratio of the parent compound to the active metabolite. Nonetheless, the combined level may be similar at equivalent doses. This ratio of parent compound to metabolite may be associated with different clinical responses. If true, this could have important clinical implications. We predict that the genotypes associated with little or no activity of the CYP2D6 enzyme will show the highest drug level of the parent compound 
(corrected for $\mathrm{mg} / \mathrm{kg}$ ). Additional exploratory analyses will include whether the trough level of the parent compound, the active metabolite, or the combination are associated with positive response or specific side effects such as sedation and/or weight gain.

\section{Four-Month Extension Study}

Several recent reviews have noted the limits of current knowledge regarding the impact of long-term drug exposure on the developing brain (Vitiello 1998) and the paucity of data on the effectiveness of pharmacotherapy in children beyond short-term trials (Vitiello and Jensen 1997). Results from recent case-control studies with risperidone suggest that adverse effects, such as weight gain, may not be evident in a short-term course of treatment (Kelly et al. 1998; Martin et al. 2000). Thus, the primary aims of the 4-month extension phase were to determine whether the therapeutic gains achieved in the short term were stable and whether adverse effects emerged or intensified over time. To meet these goals, children and adolescents classified as responders to risperidone in phase 1 (the double-blind phase or the 8-week open-label study for the placebo nonresponders) were invited to participate in the 4-month, open-label extension phase. The prospective design of the extension protocol permitted evaluation of both continued benefit and the emergence of new side effects in a systematic fashion. If risperidone demonstrated superiority to placebo in the short term as well as enduring benefit over the additional 4-month observation period without the emergence of clinically meaningful adverse effects, it would be an important addition to the available treatments of children and adolescents with autism and related disorders.

\section{Double-Blind Placebo-Controlled Withdrawal}

Subjects who showed a positive response to risperidone through the 4-month extension phase were randomly assigned to continue active medication at the same maintenance dose or to gradual withdrawal to placebo over a 3-week period. The rate of medication withdrawal was roughly 25\% per week. The maximum observation period in this final phase of the study was 8 weeks. Because withdrawal from active medication could result in relapse prior to the end of the planned observation, this design decision raised an important ethical concern that had to be balanced with scientific and clinical considerations.

First, we had to define relapse. Specific criteria for relapse were enumerated such that the return of target symptoms constituted treatment failure and marked the end of the discontinuation phase for that subject. Treating relapse as a discrete event permitted the use of clinically relevant statistical techniques that evaluate both the rate and time to relapse in each treatment group (see Analytic section below). This design feature also ensured that the placebo-controlled withdrawal period would not be prolonged to the full 8 weeks in the case of symptomatic relapse. Using the scores at randomization into the blinded discontinuation trial as baseline, relapse was defined as follows: a $25 \%$ or greater increase on the Irritability subscale of the Aberrant Behavior Checklist and two consecutive ratings by the blinded clinical evaluator of much worse or very much worse on the Global Improvement item of the CGI.

Second, although risperidone appears to have a better side effect profile than halperidol, the types and severity of adverse effects in children and adolescents have not been well documented. Moreover, virtually nothing is known about withdrawal effects such as dyskinesias, which have been shown to be common with haloperidol (Campbell et al. 1997). Thus, it was important to determine whether such withdrawal effects would also be present after treatment with risperidone. Given the chronic nature of autism and related disorders, this information should assist clinicians and families in making informed decisions about the risks and benefits of 
risperidone treatment. If subjects randomized to placebo substitution manifested relapse without emergence of dyskinesias, this would provide further evidence for the effectiveness of risperidone in this population.

Third, although autism is a chronic disorder, the symptom picture may change. New patterns of behavior may emerge over time. Thus, relapse was not a certainty. If subjects randomized to placebo substitution showed a return of target symptoms, long-term treatment with risperidone may be justified. On the other hand, if relapse did not occur, this would suggest that patients need not be exposed to a potent pharmacological agent beyond 6 months of treatment.

Fourth, drug withdrawal is common in clinical practice. Even in serious disorders such as autism, parents and/or clinicians may wonder if the target symptoms that prompted drug treatment are still present and often elect to discontinue the drug. Thus, the placebo-controlled withdrawal phase mimics clinical practice. A potentially important difference between clinical practice and the placebo-controlled withdrawal study is that subjects were carefully monitored during the withdrawal, providing the opportunity to produce generalizable data about withdrawal effects.

Fifth, if the target symptoms did return in the placebo substitution phase, we presumed that symptomatic control could be recaptured upon reinitiating active treatment. Furthermore, there is no evidence that, if relapse occurs in the short term during placebo substitution, the course of autism will be altered or worsened in the long term.

\section{Analytic plan and stopping rule for placebo discontinuation phase}

Relapse is the event of primary interest. Thus, the primary analytic strategy is to compare the rate of relapse in each group using a chi-square statistic (Fleiss 1986). Time to relapse will be evaluated for the risperidone and placebo groups by using Kaplan-Meier survival curves (logrank test). Other planned secondary analyses include the other subscales of the Aberrant Behavior Checklist, the Simpson-Angus scale, and the Abnormal Involuntary Movement Scale. Differences between the risperidone and placebo groups can be evaluated using standard linear statistical models.

In addition to the stopping rule for individual subjects who relapsed, we also developed a stopping rule for the entire discontinuation phase of the study. The stopping rule for this phase of the study was based on a simple statistical test: if the rate of relapse in the placebo group versus the active treatment group reached significance (defined as power of $80 \%$ and $p<0.01$ ) in the planned interim analyses, the discontinuation phase of the study would be stopped. These interim analyses were planned when the sample size reached 16 (8 per group) and 32 (16 per group)roughly equivalent to one third and two thirds of the expected total sample, respectively. Table 4 presents a range of values comparing the proportion of relapse across sample sizes.

\section{Use of concomitant medications}

To be eligible for the study, subjects had to be free of psychotropic medication for at least 2 weeks (4 weeks in the case of depot neuroleptics or fluoxetine). Anticonvulsant medications used for seizure control were permitted if the dose had been stable for 1 month and the subject had been seizure free for 6 months. We also needed a policy to manage subjects on stable doses of nonpsychotropic medication prior to entry. Moreover, given the long-term nature of the study, the likelihood that study participants would need concomitant medications for routine intercurrent illness or recrudescence of mild chronic conditions was predictably high.

Concomitant medications taken during the study could have pharmacokinetic interaction with 
risperidone (e.g., absorption or hepatic metabolism) and/or pharmacodynamic interaction (e.g., effects on central dopaminergic systems). Thus, when describing the purpose and design of the study to subjects and their families, investigators emphasized the importance of reviewing all ongoing or proposed medication with the research team.

To guide the use of concomitant medications during the study, we classified common pediatric medications as acceptable, drugs to avoid, and drugs considered unacceptable. The rationale for these recommendations was to prevent drug interactions that might pose a safety hazard, cause additive side effects, or confound the interpretation of behavioral response to the study medication. For example, because erythromycin or ceterazine can increase the QT interval (although presumably rare, this has also been reported for risperidone), these drugs were classified as unacceptable. Antihistamines, which may have sedative side effects, and metoclopromide, which may cause akathisia, were also considered unacceptable. The decongestant, pseudoephedrine, which may produce hyperactivity in some children, was classified as a medication to avoid. Also considered were drugs that may have an impact on risperidone concentration (e.g., tetracycline may induce CYP 2D6, which presumably would lower risperidone level).

\begin{tabular}{|c|c|c|c|c|c|}
\hline \multirow[b]{2}{*}{$\begin{array}{l}\text { Sample size } \\
\text { (per group) }\end{array}$} & \multicolumn{2}{|c|}{ Relapse-placebo } & \multicolumn{2}{|c|}{ Relapse-active } & \multirow[b]{2}{*}{$\begin{array}{c}\text { Power }^{\mathrm{a}} \\
\alpha=0.01\end{array}$} \\
\hline & $\bar{n}$ & $(\%)$ & $\mathrm{n}$ & $(\%)$ & \\
\hline 16 (8) & 6 & (75) & 1 & (12.5) & 0.47 \\
\hline $16(8)$ & 7 & (87.5) & 1 & (12.5) & 0.74 \\
\hline $16(8)$ & 8 & (100) & 2 & (25) & 0.77 \\
\hline $16(8)$ & 8 & $(100)$ & 1 & (12.5) & $0.97^{\mathrm{b}}$ \\
\hline $32(16)$ & 10 & $(62.5)$ & 2 & $(12.5)$ & 0.66 \\
\hline $32(16)$ & 10 & (62.5) & 1 & $(6.7)$ & $0.82^{\mathrm{b}}$ \\
\hline $32(16)$ & 12 & (75) & 2 & (12.5) & $0.90^{\mathrm{b}}$ \\
\hline $32(16)$ & 13 & (81.3) & 3 & (18.8) & $0.89^{\mathrm{b}}$ \\
\hline $32(16)$ & 14 & (87.5) & 5 & (31.3) & $0.79^{\mathrm{b}}$ \\
\hline $32(16)$ & 15 & (93.8) & 6 & (37.5) & $0.83^{\mathrm{b}}$ \\
\hline $50(25)$ & 15 & $(60)$ & 4 & (16) & 0.76 \\
\hline $50(25)$ & 15 & (60) & 3 & (12) & 0.87 \\
\hline $50(25)$ & 16 & (64) & 4 & (16) & 0.85 \\
\hline $50(25)$ & 18 & (72) & 6 & (24) & 0.83 \\
\hline $50(25)$ & 20 & (80) & 8 & (32) & 0.83 \\
\hline $50(25)$ & 22 & (88) & 9 & (36) & 0.92 \\
\hline
\end{tabular}

Table 4. Power Calculations for Detecting a Significant Difference in the Proportion of Relapse in Maintenance treatment Group Versus Placebo Substitution Group Across a Range of Sample Sizes

${ }^{\text {a } C a l c u l a t e d ~ w i t h ~ B o r e n s t e i n ~ e t ~ a l . ~(1997) ~ P o w e r ~ P r e c i s i o n ~ p r o g r a m ; ~ p e r c e n t a g e s ~ a r e ~ r o u n d e d ~ t o ~} 1$ decimal place (e.g., $18.75=18.8$ ).

${ }^{\mathrm{b}}$ Represents scenarios for meeting stopping rule.

These recommendations were organized into a list for investigators and a list for families. These lists were not exhaustive, but they contained specific recommendations about common medications, including over-the-counter remedies. Investigators were encouraged to consult with the subject's pediatrician about medications that were not on the list. Medication questions were also reviewed on the weekly investigator conference calls. (Copies of the Concomitant Medication guide for investigators and the handout for parents are available upon request to Larry Scahill.) 


\section{Consent and Other Ethical Issues}

The inclusion of nonverbal, often mentally retarded subjects in the study had important implications for the informed consent and assent procedures. For example, the placebocontrolled design raised questions about competence to provide consent. Current guidelines from the Department of Health and Human Services indicate that consent is a legal concept involving the capacity to make decisions about one's own life. This legal capacity begins at the age of majority (18 years of age in most states) and, in ordinary circumstances, cannot be delegated to others. When a parent gives permission for a minor, it is deemed "proxy consent." In such cases, assent is obtained to indicate affirmation by the minor. If the minor is not capable of giving assent, parents can give permission for participation in research that confers direct benefit to the child or poses only minimal risk. If the person is not competent to consent after reaching the age of majority, a guardian is needed to give permission for participation in research. Although guardianship laws vary from state to state, a subject who turns 18 during the protocol could be in an uncertain status with respect to granting informed consent. To avoid this uncertainty, we decided to exclude adolescents older than 17 years 2 months to ensure that no subject would turn 18 during the protocol.

Another ethical consideration is that children with autistic disorder accompanied by aggressive and/or SIB would likely be on medication prior to study entry. For patients deriving partial benefit from medication prior to starting the study, there was a potential for worsening of target symptoms during the required 2- to 4-week washout period. This matter had to be discussed in detail with parents and required close monitoring during the withdrawal period prior to randomization. In keeping with the fundamental ethical principle of beneficence, no subject could be withdrawn from a currently effective medication. Finally, given the complexity of the study design, we evaluated parental impressions of the consent procedures in a 17-item questionnaire developed for the study.

\section{Clinical implications}

Autistic disorder is a severe developmental disorder of early childhood onset that can be accompanied by behavioral problems such as tantrums, SIB, aggression, and agitation. Although several medications are used in the treatment of children and adolescents with autistic disorder, there is no commonly accepted standard treatment. Therefore, treatment focuses on target symptoms that may be amenable to pharmacological intervention. The RUPP autism study (with the initial double-blind, placebo-controlled phase, the open-label extension phase followed by the placebo-substitution phase) was designed to evaluate short-term efficacy and safety as well as the long-term stability and safety of risperidone for aggression, SIB, and/or agitation that is consistent with this clinical model. The results of the study are likely to provide guidance to clinicians and families on the use of risperidone in the treatment of children and adolescents with autistic disorder and these target symptoms.

\section{Acknowledgments}

The authors acknowledge the efforts of Allison Lancor and Abigail Newton in preparing the original manuscript; the contribution of Greg Humma, Rh.P., for implementation of the medication program; James Robinson, M.Ed., and colleagues for data management; Jocelyn Atienza, M.S., for managing DNA samples; and Dr. Jane Nofer for training investigators on the Autism Diagnostic Interview. We also acknowledge the comments and guidance of the NIMH Data and Safety Monitoring Board and the RUPP Scientific Advisory Board.

Research support came from NIMH Contract N01MH70001 (Indiana); N01MH80011 (Ohio State); N01MH70010 (UCLA); N0MH70009 (Yale); NIH Clinical Research Center grants M01-RR00052 (Johns Hopkins), M01-RR00750 (Indiana), M01-RR00034 (Ohio State), M01-RR00865 (UCLA), M01-RR06022 (Yale), and K23 
MH01966 (Dr. McGough); K01 MH0179201 (Dr. Martin) K24 (Dr. McCracken); and the Korczak Foundation (Dr. Scahill). Study medications were donated by Janssen Pharmaceuticals.

The opinions and assertions contained in this report are the private views of the authors and are not to be construed as official or as reflecting the views of the Department of Health and Human Services or the National Institutes of Health.

\section{References}

Aman MG, Singh NN: Aberrant Behavior Checklist Manual. East Aurora (New York), Slossan Educational Publications, 1986.

Aman MG, Singh NN, Stewart AW, Field CJ: The Aberrant Behavior Checklist: A behavior rating scale for the assessment of treatment effects. Am J Ment Defic 89(5):485-491, 1985.

Aman MG, Van Bourgondien ME, Wolford PL, Sarphare G: Psychotropic and anticonvulsant drugs in subjects with autism: Prevalence and patterns of use. J Am Acad Child Adolesc Psychiatry 34(2):1672-1681, 1995.

Anderson LT, Campbell M, Adams P, Small AM, Perry R, Shell J: The effects of haloperidol on discrimination learning and behavioral symptoms in autistic children. J Autism Dev Disord 19(2):277-239, 1989.

Arnold LE, Aman MG, Martin A, Collier-Crespin A, Vitiello B, Tierney E, Asarnow R, Bell-Bradshaw F, Freeman BJ, Gates-Ulanet P, Klin A, McCracken JT, McDougle CJ, McGough JJ, Posey DJ, Scahill L, Swiezy NB, Ritz L, Volkmar F: Assessment in multisite randomized clinical trials of patients with autistic disorder: The Autism RUPP Network. Research units on Pediatric Psychopharmacology. J Autism Dev Disord 30(2):99_ 111, 2000.

Borenstein M, Rothstein H, Cohen J: Power and Precision. Mahwah (New Jersey), Erlbaum, 1997.

Campbell M, Armenteros JL, Malone RP, Adams PB, Eisenberg ZW, Overall JE: Neuroleptic-related dyskinesias in autistic children: A prospective, longitudinal study. J Am Acad Child Adolesc Psychiatry 36(6):835-843, 1997.

Chakrabarti S, Fombonne E: Pervasive developmental disorders in pre-school children. JAMA 285:3093-3099, 2001.

Cohen DJ, Volkmar FR: Handbook of Autism and Pervasive Developmental Disorders, 2nd ed. New York, Wiley, 1997.

Fleiss JL: The Design and Analysis of Clinical Experiments. New York, Wiley, 1986.

Fombonne E: The epidemiology of autism: A review. Psychol Med 29(4):769-786, 1999.

Gibbons RD, Hedeker D, Elkin I, Waternaux C, Kraemer HC, Greenhouse JB, Shea MT, Imber SD, Sotsky SM, Watkins JT: Some conceptual and statistical issues in analysis of longitudinal psychiatric data. Application to the NIMH treatment of Depression Collaborative Research Program dataset. Arch Gen Psychiatry 50:739-750, 1993.

He N, Daniel HI, Hajiloo L, Shockley D: Dextromethorphan O-demethylation polymorphism in an AfricanAmerican population. Eur J Clin Pharmacol 55(6):457-459, 1999.

Heykants J, Huang ML, Mannens G, Meuldermans W, Snoeck E, Van Beijsterveldt L, Van Peer A, Woestenborghs R: The pharmacokinetics of risperidone in humans: A summary. J Clin Psychiatry 55(Suppl):13-17, 1994.

Kumra S, Herion D, Jacobsen LK, Briguglia C, Grothe D: Case study: Risperidone-induced hepatotoxicity in pediatric patients. J Am Acad Child Adolesc Psychiatry 36(5):701-705, 1997.

Lord C, Rutter M, Le Couteur A: Autism Diagnostic Interview-Revised: A revised version of a diagnostic interview for caregivers of individuals with possible pervasive developmental disorders. J Autism Dev Disord 24(5):659-685, 1994.

Martin A, Landau J, Leebens P, Ulizio K, Cicchetti D, Scahill L, Leckman JF: Risperidone-associated weight gain in children and adolescents: A retrospective chart review. J Child Adoles Psychopharmacol 10:259-268, 2000.

Mashburn EC, Aman MG: Factor validity and norms for the Abberant Behavior Checklist in a community sample of children with mental retardation. J Autism Dev Disord 22(3):357-373, 1992.

McDougle CJ, Scahill L, McCracken JT, Aman MT, Tierney E, Arnold LE, Freeman BJ, Martin A, McGough JJ, Cronin P, Posey DJ, Riddle MA, Ritz L, Sweizy NB, Vitiello B, Volkmar FR, Votolato NA, Walson P: Research Units on Pediatric Psychopharmacology (RUPP) Autism Network. Background and rationale for an initial controlled study of risperidone [Review]. Child Adolesc Psychiatr Clin N Am 9(1):201-224, 2000.

Poolsup N, Li Wan Po A, Knight TL: Pharmacogenetics and pharmacotherapy. J Clin Pharm Ther 25(3):197-200, 2000. 
Sandler AD, Sutton KA, DeWeese J, Girardi MA, Sheppard V, Bodfish JW: Lack of benefit of a single dose of synthetic human secretin in the treatment of autism and pervasive developmental disorder. N Engl J Med 341(24):1801-1806, 1999.

Vitiello B: Pediatric psychopharmacology and the interaction between drugs and the developing brain [Review]. Can J Psychiatry 43(6):582-584, 1998.

Vitiello B, Jensen PS: Medication development and testing in children and adolescents: Current problems, future directions. Arch Gen Psychiatry 54:871-876, 1997. 